Published in final edited form as:

Curr Opin Cardiol. 2018 May ; 33(3): 290-297. doi:10.1097/HCO.0000000000000510.

\title{
Extracellular vesicles in cardiovascular homeostasis and disease
}

\author{
Joshua D. Hutcheson ${ }^{1}$ and Elena Aikawa ${ }^{2,3}$ \\ 1Department of Biomedical Engineering, Florida International University, Miami, FL, 33174, USA \\ ${ }^{2}$ Center for Interdisciplinary Cardiovascular Sciences, Brigham and Women's Hospital, Boston, \\ MA, 02115, USA \\ ${ }^{3}$ Cardiovascular Division, Department of Medicine, Center for Excellence in Vascular Biology, \\ Brigham and Women's Hospital, Harvard Medical School, Boston, MA, 02115, USA
}

\begin{abstract}
Purpose of review-Extracellular vesicles (EVs) have emerged as one of the most important means through which cells interact with each other and the extracellular environment, but EV research remains challenging due to their small size and limited amount of material required for traditional molecular biology assays. The advent of new technologies and standards in the field, however, have led to increased mechanistic insight into EV function. Herein, the latest studies on the role of EVs in cardiovascular physiology and pathology are discussed.
\end{abstract}

Recent findings-EVs help control cardiovascular homeostasis and remodeling by mediating communication between cells and directing alterations in the extracellular matrix to respond to changes in the environment. The message carried from the parent cell to extracellular space can be intended for both local (within the same tissue) and distal (downstream of blood flow) targets. Pathological cargo loaded within EVs could further result in various diseases. On the other hand, new studies indicate that injection of EVs obtained from cultured cells into diseased tissues can promote restoration of normal tissue function.

Summary-EVs are an integral part of cell and tissue function, and harnessing the properties inherent to EVs may provide a therapeutic strategy to promote tissue regeneration.

\section{Keywords}

extracellular vesicles; exosomes; microvesicles; microparticles; cardiovascular disease

\section{Introduction}

\begin{abstract}
Vesicles secreted from cells into the extracellular space (extracellular vesicles or EVs) play an increasingly recognized role in both physiological and pathological processes. Once
\end{abstract}

Correspondence: Elena Aikawa, MD, PhD, Brigham and Women's Hospital, Harvard Medical School, 77 Avenue Louis Pasteur, NRB 741, Boston, MA, 02115, Phone: 617-730-7755, Fax: 617-730-7791, eaikawa @ bwh.harvard.edu; or Joshua D. Hutcheson, PhD, Department of Biomedical Engineering, Florida International University, 10555 W Flagler St., EC 2612, Miami, FL 33174, Phone: 305-348-0157, Fax: 305-348-6954, jhutches@ fiu.edu.

Conflict of interest: None. 
thought to serve solely as a mechanism to remove waste material from cells, studies conducted over the past two decades have demonstrated that EVs allow cells to interact with their neighbors and the extracellular environment. EVs contain genetic (RNA and DNA) and proteomic material. Importantly, while the proteins and genetic material may reflect properties of the parent cells, EVs are generally not mirror images of the cellular membrane. This suggests that EVs are derived from specific subcellular locations, and the contents are likely intended for a defined function in the extracellular space.

Due to their small size, however, difficulties remain in characterizing the myriad of EVs released by cells. At any given time, cells may shed EVs through direct budding at the plasma membrane or through a variety of intracellular trafficking pathways that result in fusion of multivesicular bodies to the membrane and subsequent expulsion of intraluminal vesicles (Figure 1). Multivesicular bodies form within cells following invagination of plasma membrane in endocytotic vesicles. As these recycling endosomes traffic through the cell cytoplasm, new vesicles form through invagination of small vesicles into the intraluminal space of the larger endosome. The resultant EVs released by membrane fusion of the endosome are usually termed exosomes. EVs that bud from the membrane require coordinated cytoskeletal dynamics that lead to plasma membrane protrusion, and actinmediated constriction that separates the membrane and releases the EV into the extracellular space. These EVs are often termed microvesicles or microparticles, and generally have a larger average diameter than EVs produced through intracellular trafficking. These canonical mechanisms of EV formation, however, may not capture all forms of EV genesis, namely those derived from intracellular organelles.

The lack of reliable methods to separate EV populations post-release leads to confusion surrounding overlap in EV functions across numerous studies. Investigators often use specific names to describe the function or properties of the EV populations of interest (e.g., calcifying matrix vesicles, tolerosomes, prostasomes), but it is unclear whether these different functions belong to the same EVs or different populations of EVs. Even more general terms such as microvesicles, microparticles, and exosomes have overlapping characteristics (e.g., size ranges, sedimentation properties, proteomic markers). Therefore, studies often use a combination of factors such as EV size and known protein markers to verify the presence of specific EV populations. Though different EV populations may contain different membrane lipid profiles, membrane-associated proteins, and intraluminal genetic material and proteins, the relative abundance of these factors per EV is relatively small and difficult to assay.

Barriers remain in characterizing EVs, but new technologies such as tunable resistive pulse sensing [1] and nanoparticle tracking [2] have emerged as reliable methods to measure EV size, concentration, and charge. Traditionally, EV characterization relied upon electron microscopy to provide the requisite resolution for single EV analyses. However, these imaging-based analyses are low-throughput and not easily amenable to determination of EV contents. Adaptation of flow cytometric protocols has allowed for measurement of proteins in individual EVs and identification of separate EV populations [3*]. Poor signal-to-noise ratios remain a problem in these measurements, limiting its ability to identify low abundance proteins. The development of new methods to measure EV properties and their subcellular 
origins will likely lead to needed mechanistic insight. Excellent reviews have focused on the fingerprinting and functional characterization of various EV populations. To broadly characterize the role of EVs in cardiovascular homeostasis and disease, we will discuss EV function within two larger contexts: 1) EVs as mediators of intracellular communication and 2) EVs as mediators of extracellular matrix (ECM) remodeling. The reader should note, however, that in some cases these functions could belong to the same EVs. Finally, we will briefly review efforts to harness EV properties for diagnostic and therapeutic purposes.

\section{Extracellular Vesicles as Mediators of Intracellular Communication}

The ability to serve as vehicles of intercellular communication is one of the most wellstudied functions of EVs; however, due to the myriad of EV subtypes and equally diverse set of cargo, the mechanisms by which messages are selectively delivered from source cells to target cells remain the subject of ongoing research. In accordance with previous observations with synthetic nanoparticles used for intracellular delivery, EV size plays an important role in the ability to transfer material (e.g., RNA or proteins) into a target cell. Relatively smaller EVs $(30-50 \mathrm{~nm})$ tend to exhibit higher uptake in target cells and consequently induce larger phenotypic responses [4]. Changes in the extracellular environment can influence both EV physical properties and cargo. Elevated glucose to diabetic levels induces an increase in both the rate of EV release and the size of EVs produced by endothelial cells in culture [5]. Circulating endothelial-derived EVs in patients with cerebrovascular atherosclerosis exhibit altered protein expression consistent with known changes in endothelial cell phenotype [6], and vascular smooth muscle cells (VSMCs) exposed to atherogenic lipoproteins exhibit altered EV microRNA profiles [7**]. These changes in EV size and/or cargo could alter communication between cells to respond to an extracellular cue and may exacerbate pathological conditions by disrupting homeostatic paracrine signaling.

EV-mediated paracrine signaling can act both locally (i.e., between cells within the same tissue) or distally by traveling with blood to downstream targets (Figure 2). Arteriosclerosis, vascular restenosis following catheter-based interventions, and outward aneurysmal remodeling involve phenotypic changes in vascular endothelial and smooth muscle cells (SMCs). Accumulating evidence suggests a role for EVs in mediating the cellular crosstalk in response to extracellular stimuli that trigger changes in phenotype and the resultant tissue remodeling. Vascular SMC proliferation and migration from the medial space is often an early response to pathological cues. Patients with coronary artery disease exhibit an inverse relationship between percutaneous coronary intervention rate and the expression of microRNA-126 in circulating EVs [8]. Intravenous injection of endothelial-derived EVs loaded with microRNA-126 prevents SMC proliferation, migration, and neointima formation in a mouse model of vascular injury. Similarly, platelet-derived EVs can induce a proinflammatory remodeling phenotype in SMCs, including increased migration and proliferation [9]. Systemic pressures (e.g., introduction of pathological agents) often first effect blood cells and the endothelial cells that line the blood vessel. These studies suggest that EVs can mediate communication from these cells to the SMCs located deeper in the tissue. In turn, vascular SMCs directly control tissue homeostasis through synthesis and degradation of extracellular matrix components. 
EV-mediated signaling, however, is not unidirectional from the blood into the vascular wall. Endoplasmic reticulum stress leads to production of SMC-derived EVs, which promote endothelial cell dysfunction in a model of thoracic aortic aneurysm [10]. Classically, endothelial cell dysfunction leads to expression of adhesion proteins that aid in the translocation of leukocytes from the blood into vascular tissue, wherein these cells contribute to vascular wall remodeling. Following acute myocardial infarction, large numbers splenic monocytes mobilize to the damaged myocardium. Recent evidence suggests a role for endothelial-derived EVs in the initial monocytic recruitment [11*]. The total number of circulating EVs increases in plasma following myocardial infarction in both mice and humans, and the elevated number of EVs exhibit protein markers that suggest an endothelial cell origin. These EVs also contain the same microRNA-126 that modulates VSMC phenotype. Accumulation of these EVs in the spleen promotes mobilization of monocytes, which migrate to the infarcted site and aid in tissue repair. Thus, endothelial cell-derived EVs containing microRNA-126 can affect tissue homeostasis through both local regulation of vascular SMC phenotype and distal recruitment of leukocytes.

EVs as mediators of inter-organ communication has received increased attention due to pioneering cancer research [12]. EVs carry tumorigenic components through systemic circulation and can prime distal sites for metastatic invasion. Recent studies suggest that adipose tissue serves as a source for a large number of circulating EVs [13**]. Knocking out Dicer, the microRNA processing enzyme, in mouse adipose tissue leads to decreased glucose tolerance. Reintroducing adipose tissue containing Dicer into these mice improves glucose tolerance and decreases circulating levels of insulin and liver-derived fibroblast growth factor-21 (FGF21). These findings show the importance of adipose tissue in regulating gene expression throughout the body and underscore the role of EVs in systemic homeostasis.

\section{Extracellular Vesicles as Mediators of Cell-Matrix Interactions}

ECM structure determines the biomechanical function of cardiovascular tissues, and inappropriate remodeling contributes to morbidity and mortality. Failure of the collagenous fibrous cap contributes to atherosclerotic plaque rupture, the leading cause of death globally. Typically, plaque vulnerability associates with low collagen content in the fibrous cap, which compromises its tensile strength [14]. Recent computational studies, however, highlight the presence of destabilizing microcalcifications (approximately $5 \mu \mathrm{m}$ ), smaller than the resolution limits of traditional clinical imaging modalities, in the cap of "vulnerable plaques" as a determinant of their biomechanical failure $[15,16]$. Prospective clinical data from the Multi-Ethnic Study of Atherosclerosis (MESA) trial [17], corroborates the biomechanical model predictions linking microcalcifications with plaque rupture. These data indicate that larger calcifications may stabilize atherosclerotic plaques, whereas "spotty" calcification (or early microcalcificaiton) contributes to plaque destabilization [18]. Though large calcifications may stabilize plaques in the short-term, continued calcification growth can also induce vessel stenosis, reduced blood flow, and elevated systemic resistance [19]. Therefore, calcification morphology correlates with cardiovascular risk. 
Calcification comprises EVs that serve as nucleating foci for mineralization. Yet, the process by which EVs contribute to vascular calcification is difficult to study due to the inability to visualize and monitor early calcification events in vivo. We recently developed a 3dimensional in vitro platform in which we seeded EVs obtained from human vascular SMCs in a collagen hydrogel [20*]. This platform allowed us to directly observe the growth of microcalcification from single EVs to mature calcifications spectroscopically similar to those observed in human atherosclerotic plaques. Using this platform and analysis of human plaques, we documented an inverse relationship between collagen and microcalcification size. Calcifying EVs aggregate and fuse to form microcalcifications, and when unencumbered by collagen, the microcalcifications serve as building blocks for larger calcifications (Figure 3).

The formation of calcifying EVs begins with phenotypic changes within the parent cells that result in enrichment of pro-mineralizing factors. In valve interstitial cells, elevated extracellular calcium and phosphate induce release of EVs enriched with Annexin VI [21*]. Similar to its function in vascular SMCs, Annexin VI mediates calcium binding in EVs, promoting mineral formation. Conditions that simulate inflammation-induced osteogenic changes in SMCs result in the loading of active tissue non-specific alkaline phosphatase (TNAP) into EVs through intracellular trafficking mechanisms directed by the sorting receptor sortilin [22*]. Active TNAP hydrolyzes the calcification inhibitor pyrophosphate and produces free phosphate required for mineralization, promoting mineralization even in normal ranges of extracellular phosphate. Vascular SMC phenotypic changes and resultant production of calcifying EVs also depends on feedback from the ECM. Impaired collagen sensing through the collagen receptor discoidin domain receptor-1 (DDR-1) leads to an increase in fibrotic collagen and calcifying EV release from SMCs [23], suggesting that the EVs can serve as a mechanism for delivery of ECM and mineral components to help the vascular SMCs respond to lost extracellular homeostasis. The altered loading and trafficking patterns that result in the formation of calcifying EVs offer potential therapeutic targets for cardiovascular calcification and vascular wall remodeling, major contributors to morbidity with no known treatment.

EVs also play a significant role in post-plaque rupture responses. Immediately following atherosclerotic plaque rupture leads to thrombotic vessel occlusion associated with platelet activation and activation of the coagulation cascade. Progression of the coagulation cascade requires the presence and activation of several enzymes that aid in the generation of thrombin, and many of the rate limiting enzymatic reactions begin at the surface of EVs [24]. Tissue factor, a key enzyme that initiates the coagulation cascade, is found on subpopulations of EVs derived from vascular SMCs [25*], endothelial cells [26], monocytes/macrophages [27], and platelets [28]. Further, the charged phospholipid profile (e.g., enrichment of phosphatidylserine) characteristic of many EV populations may also aid in the generation of thrombin [28]. In response to the generation of thrombin, local platelets release exosomal EVs that mediate paracrine signaling to endothelial cells [29]. In turn, the endothelial cells release EVs enriched with matrix metallopreinase-10, which exacerbate the pro-inflammatory vascular wall remodeling [30]. Acetylsalicylic acid (aspirin) treatment significantly reduces the release of exosomal EVs from platelets [29], perhaps contributing to its function in preventing thrombosis. 


\section{Extracellular Vesicles as Therapeutic Vehicles and Diagnostic Tools}

Circulating EVs are often analyzed for their potential to serve as diagnostic tools for a variety of pathologies. Since EV contents can change in a manner that reflects alterations in cell phenotype, EVs in the blood stream can serve as a non-invasive means to measure the phenotypic state of tissues throughout the body. For example, SMCs exposed to atherogenic lipoproteins release EVs with significantly altered microRNA profiles, including a decrease in microRNA-24-3p and -130a-3p [7**]. Perhaps relatedly, these two microRNAs exhibit decreased expression in circulating EVs from individuals with familial hypercholesterolemia. In addition to their diagnostic potential, however, recent studies have shown a tremendous capacity for EVs to serve as therapeutic agents and restore tissue homeostasis [31]. Endothelial EVs can carry function endothelial nitric oxide synthase (eNOS), which works in an autocrine manner to restore homeostasis following fatty acidinduced oxidative stress [32]. In a similar manner, introduction of EVs obtained from cultured cells can prevent or reverse pathological remodeling in cardiovascular tissues. EVs from cardiosphere-derived cells can reduce myocardial wall damage when injected into the coronary arteries of rat and pig models of acute myocardial infarction [33**]. The cardioprotection conferred by these EVs is not observed when fibroblast EVs are injected into the same models, indicating the specificity of the effect. Stem cell-derived EVs have also shown remarkable cardioprotective and regenerative potential [34]. The reparative capacity of these EVs is likely conferred by efficient delivery of microRNAs that promote homeostatic gene expression and non-inflammatory phenotypes in diseased tissue [35]. Given their natural function in delivering cargo between cells, EVs can overcome the barriers often faced effective drug and therapeutic delivery.

\section{Conclusion}

Harnessing the inherent delivery properties associated with EVs, either through culture and collection of sufficient numbers of cellular-derived EVs or synthetic mimics, could revolutionize the delivery of therapeutics, allowing for the translation of strategies (e.g., nucleic acid-based therapies) that cannot easily reach intracellular targets. Special consideration, however, should be given to potential effects on systemic homeostasis and unintended uptake in off-target tissues. The continued development of new tools to characterize and quantify EVs will also help this effort by filling lingering knowledge gaps on $\mathrm{EV}$ properties that mediate function. Once thought to exist solely as a mechanism to dispose of unwanted cellular material, it is now clear that EVs play relevant and diverse roles in cell biology, including cardiovascular physiology and disease (Table 1). This relatively young field may only be at the beginning of uncovering the importance of EVs in physiology and pathology.

\section{Acknowledgments}

Funding: Dr. Elena Aikawa is supported by National Institutes of Health (NIH) grants R01HL 114805 and R01HL 136431 and Harvard Catalyst Big Ideas, Small Features: Advanced Microscopic and Nanoscale Technologies Pilot Award. Dr. Joshua Hutcheson is supported by a Scientist Development Grant from the American Heart Association (17SDG633670259). 


\section{References}

1. Vogel R, Coumans FA, Maltesen RG, Boing AN, Bonnington KE, Broekman ML, Broom MF, Buzas EI, Christiansen G, Hajji N, et al. A standardized method to determine the concentration of extracellular vesicles using tunable resistive pulse sensing. J Extracell Vesicles. 2016; 5:31242. [PubMed: 27680301]

2. Parsons MEM, McParland D, Szklanna PB, Guang MHZ, O'Connell K, O'Connor HD, McGuigan C, Ni Ainle F, McCann A, Maguire PB. A Protocol for Improved Precision and Increased Confidence in Nanoparticle Tracking Analysis Concentration Measurements between 50 and 120 nm in Biological Fluids. Front Cardiovasc Med. 2017; 4:68. [PubMed: 29164135]

3*. van der Pol E, de Rond L, Coumans FAW, Gool EL, Boing AN, Sturk A, Nieuwland R, van Leeuwen TG. Absolute sizing and label-free identification of extracellular vesicles by flow cytometry. Nanomedicine. 2018 This study provides a valuable technique to characterize and quantify individual EV populations using common flow cytometry techniques.

4. Caponnetto F, Manini I, Skrap M, Palmai-Pallag T, Di Loreto C, Beltrami AP, Cesselli D, Ferrari E. Size-dependent cellular uptake of exosomes. Nanomedicine. 2017; 13:1011-1020. [PubMed: 27993726]

5. Burger D, Turner M, Xiao F, Munkonda MN, Akbari S, Burns KD. High glucose increases the formation and pro-oxidative activity of endothelial microparticles. Diabetologia. 2017; 60:17911800. [PubMed: 28601907]

6. Goetzl EJ, Schwartz JB, Mustapic M, Lobach IV, Daneman R, Abner EL, Jicha GA. Altered cargo proteins of human plasma endothelial cell-derived exosomes in atherosclerotic cerebrovascular disease. FASEB J. 2017; 31:3689-3694. [PubMed: 28476896]

7**. de Gonzalo-Calvo D, Cenarro A, Garlaschelli K, Pellegatta F, Vilades D, Nasarre L, CaminoLopez S, Crespo J, Carreras F, Leta R, et al. Translating the microRNA signature of microvesicles derived from human coronary artery smooth muscle cells in patients with familial hypercholesterolemia and coronary artery disease. J Mol Cell Cardiol. 2017; 106:55-67. Treating smooth muscle cells with atherogenic lipids leads to a change in EV microRNA profiles. The observation that these changes are also mirrored in circulating EVs from hypercholesterolemic patients leads to the hypothesis that EVs from the artery wall can enter systemic circulation and affect tissues distal from the plaque site. [PubMed: 28342976]

8. Jansen F, Stumpf T, Proebsting S, Franklin BS, Wenzel D, Pfeifer P, Flender A, Schmitz T, Yang X, Fleischmann BK, et al. Intercellular transfer of miR-126-3p by endothelial microparticles reduces vascular smooth muscle cell proliferation and limits neointima formation by inhibiting LRP6. J Mol Cell Cardiol. 2017; 104:43-52. [PubMed: 28143713]

9. Vajen T, Benedikter BJ, Heinzmann ACA, Vasina EM, Henskens Y, Parsons M, Maguire PB, Stassen FR, Heemskerk JWM, Schurgers LJ, et al. Platelet extracellular vesicles induce a proinflammatory smooth muscle cell phenotype. J Extracell Vesicles. 2017; 6:1322454. [PubMed: 28717419]

10. Jia LX, Zhang WM, Li TT, Liu Y, Piao CM, Ma YC, Lu Y, Wang Y, Liu TT, Qi YF, et al. ER stress dependent microparticles derived from smooth muscle cells promote endothelial dysfunction during thoracic aortic aneurysm and dissection. Clin Sci (Lond). 2017; 131:1287-1299. [PubMed: 28468950]

11*. Akbar N, Digby JE, Cahill TJ, Tavare AN, Corbin AL, Saluja S, Dawkins S, Edgar L, Rawlings $\mathrm{N}$, Ziberna K, et al. Endothelium-derived extracellular vesicles promote splenic monocyte mobilization in myocardial infarction. JCI Insight. 2017; 2 Classically, endothelial cells recruit monocytes to an infarct site through direct interaction in the blood stream, but this study shows that endothelial-derived EVs can initiate mobilization of monocytes from the spleen following a myocardial infarct.

12. O'Loghlen A. Role for extracellular vesicles in the tumour microenvironment. Philos Trans R Soc Lond B Biol Sci. 2018; 373

13**. Thomou T, Mori MA, Dreyfuss JM, Konishi M, Sakaguchi M, Wolfrum C, Rao TN, Winnay JN, Garcia-Martin R, Grinspoon SK, et al. Adipose-derived circulating miRNAs regulate gene expression in other tissues. Nature. 2017; 542:450-455. Studies often focus on local signaling mechanisms in cardiovascular remodeling and disease, but this study illustrates the importance of 
considering cross-talk between tissues in the maintenance of systemic homeostasis. Adopisederived EVs released into circulation can influence cell phenotypes and behavior across the body. [PubMed: 28199304]

14. Libby P. Collagenases and cracks in the plaque. J Clin Invest. 2013; 123:3201-3203. [PubMed: 23908120]

15. Kelly-Arnold A, Maldonado N, Laudier D, Aikawa E, Cardoso L, Weinbaum S. Revised microcalcification hypothesis for fibrous cap rupture in human coronary arteries. Proc Natl Acad Sci U S A. 2013; 110:10741-10746. [PubMed: 23733926]

16. Maldonado N, Kelly-Arnold A, Cardoso L, Weinbaum S. The explosive growth of small voids in vulnerable cap rupture; cavitation and interfacial debonding. J Biomech. 2013; 46:396-401. [PubMed: 23218838]

17. Criqui MH, Denenberg JO, Ix JH, McClelland RL, Wassel CL, Rifkin DE, Carr JJ, Budoff MJ, Allison MA. Calcium density of coronary artery plaque and risk of incident cardiovascular events. JAMA. 2014; 311:271-278. [PubMed: 24247483]

18. Hutcheson JD, Maldonado N, Aikawa E. Small entities with large impact: microcalcifications and atherosclerotic plaque vulnerability. Curr Opin Lipidol. 2014; 25:327-332. [PubMed: 25188916]

19. Ruiz JL, Hutcheson JD, Aikawa E. Cardiovascular calcification: current controversies and novel concepts. Cardiovasc Pathol. 2015; 24:207-212. [PubMed: 25797772]

20**. Hutcheson JD, Goettsch C, Bertazzo S, Maldonado N, Ruiz JL, Goh W, Yabusaki K, Faits T, Bouten C, Franck G, et al. Genesis and growth of extracellular-vesicle-derived microcalcification in atherosclerotic plaques. Nat Mater. 2016; 15:335-343. Studies on the role of EVs in calcification are limited by the inability to visualize the process from the earliest stages. This study utilized advanced microscopy techniques with synthetic 3-D in vitro platforms to establish new insight into the genesis of calcification and the formation of plaque-destabilizing microcalcifications. [PubMed: 26752654]

21*. Cui L, Rashdan NA, Zhu D, Milne EM, Ajuh P, Milne G, Helfrich MH, Lim K, Prasad S, Lerman DA, et al. End stage renal disease-induced hypercalcemia may promote aortic valve calcification via Annexin VI enrichment of valve interstitial cell derived-matrix vesicles. J Cell Physiol. 2017; 232:2985-2995. This study is the first to show that conditions associated with renal disease induce changes in EVs that can lead to aortic valve calcification. [PubMed: 28369848]

22*. Goettsch C, Hutcheson JD, Aikawa M, Iwata H, Pham T, Nykjaer A, Kjolby M, Rogers M, Michel T, Shibasaki M, et al. Sortilin mediates vascular calcification via its recruitment into extracellular vesicles. J Clin Invest. 2016; 126:1323-1336. Intracellular trafficking mechanisms play an important role in the building of EVs, and this study shows that the sortilin receptor is required for loading calcifiction-associated proteins for mineralization into calcifying EVs. [PubMed: 26950419]

23. Krohn JB, Hutcheson JD, Martinez-Martinez E, Irvin WS, Bouten CV, Bertazzo S, Bendeck MP, Aikawa E. Discoidin Domain Receptor-1 Regulates Calcific Extracellular Vesicle Release in Vascular Smooth Muscle Cell Fibrocalcific Response via Transforming Growth Factor-beta Signaling. Arterioscler Thromb Vasc Biol. 2016; 36:525-533. [PubMed: 26800565]

24. Rothmeier AS, Marchese P, Langer F, Kamikubo Y, Schaffner F, Cantor J, Ginsberg MH, Ruggeri ZM, Ruf W. Tissue Factor Prothrombotic Activity Is Regulated by Integrin-arf6 Trafficking. Arterioscler Thromb Vasc Biol. 2017; 37:1323-1331. [PubMed: 28495929]

25*. Kapustin AN, Schoppet M, Schurgers LJ, Reynolds JL, McNair R, Heiss A, Jahnen-Dechent W, Hackeng TM, Schlieper G, Harrison P, et al. Prothrombin Loading of Vascular Smooth Muscle Cell-Derived Exosomes Regulates Coagulation and Calcification. Arterioscler Thromb Vasc Biol. 2017; 37:e22-e32. Thrombus formation is thought to be mediated by platelets and endothelial cells, but this study shows that EVs from smooth muscle cells can also play an important role. [PubMed: 28104608]

26. Holnthoner W, Bonstingl C, Hromada C, Muehleder S, Zipperle J, Stojkovic S, Redl H, Wojta J, Schochl H, Grillari J, et al. Endothelial Cell-derived Extracellular Vesicles Size-dependently Exert Procoagulant Activity Detected by Thromboelastometry. Sci Rep. 2017; 7:3707. [PubMed: 28623360] 
27. Chiva-Blanch G, Laake K, Myhre P, Bratseth V, Arnesen H, Solheim S, Badimon L, Seljeflot I. Platelet-, monocyte-derived and tissue factor-carrying circulating microparticles are related to acute myocardial infarction severity. PLoS One. 2017; 12:e0172558. [PubMed: 28207887]

28. Tripisciano C, Weiss R, Eichhorn T, Spittler A, Heuser T, Fischer MB, Weber V. Different Potential of Extracellular Vesicles to Support Thrombin Generation: Contributions of Phosphatidylserine, Tissue Factor, and Cellular Origin. Sci Rep. 2017; 7:6522. [PubMed: 28747771]

29. Goetzl EJ, Goetzl L, Karliner JS, Tang N, Pulliam L. Human plasma platelet-derived exosomes: effects of aspirin. FASEB J. 2016; 30:2058-2063. [PubMed: 26873936]

30. Martinez de Lizarrondo S, Roncal C, Calvayrac O, Rodriguez C, Varo N, Purroy A, Lorente L, Rodriguez JA, Doeuvre L, Hervas-Stubbs S, et al. Synergistic effect of thrombin and CD40 ligand on endothelial matrix metalloproteinase-10 expression and microparticle generation in vitro and in vivo. Arterioscler Thromb Vasc Biol. 2012; 32:1477-1487. [PubMed: 22492089]

31. Bei Y, Das S, Rodosthenous RS, Holvoet P, Vanhaverbeke M, Monteiro MC, Monteiro VVS, Radosinska J, Bartekova M, Jansen F, et al. Extracellular Vesicles in Cardiovascular Theranostics. Theranostics. 2017; 7:4168-4182. [PubMed: 29158817]

32. Mahmoud AM, Wilkinson FL, McCarthy EM, Moreno-Martinez D, Langford-Smith A, Romero M, Duarte J, Alexander MY. Endothelial microparticles prevent lipid-induced endothelial damage via Akt/eNOS signaling and reduced oxidative stress. FASEB J. 2017; 31:4636-4648. [PubMed: 28687612]

33**. de Couto G, Gallet R, Cambier L, Jaghatspanyan E, Makkar N, Dawkins JF, Berman BP, Marban E. Exosomal MicroRNA Transfer Into Macrophages Mediates Cellular Postconditioning. Circulation. 2017; 136:200-214. Macrophage phenotype plays a major role in determinign the severity of tissue damage and regeneration of myocardium following an infarct. This study shows that injection of EVs derived from specific cardiosphere-derived cells into the infarct site results in an anti-inflammatory macrophage phenotype and a cardioprotective effect. These findings demonstrate the potential of EV-based therapeutics for cardiovascular disease. [PubMed: 28411247]

34. Willis GR, Kourembanas S, Mitsialis SA. Toward Exosome-Based Therapeutics: Isolation, Heterogeneity, and Fit-for-Purpose Potency. Front Cardiovasc Med. 2017; 4:63. [PubMed: 29062835]

35. Eirin A, Zhu XY, Puranik AS, Woollard JR, Tang H, Dasari S, Lerman A, van Wijnen AJ, Lerman LO. Integrated transcriptomic and proteomic analysis of the molecular cargo of extracellular vesicles derived from porcine adipose tissue-derived mesenchymal stem cells. PLoS One. 2017; 12:e174303. [PubMed: 28333993] 


\section{Article Summary}

- Cells rely on EVs to interact with the extracellular environment.

- EVs mediate communication between cells in the same tissue and by carrying messages through circulation to distant tissues.

- Extracellular matrix formation and remodeling often relies on the presence of specific enzymes on and within EVs.

- Delivery of EVs from cultured stem cell populations can protect and regenerate diseased cardiovascular tissues.

- Controlling and/or mimicking EV function can serve as a powerful therapeutic strategy for cardiovascular diseases. 


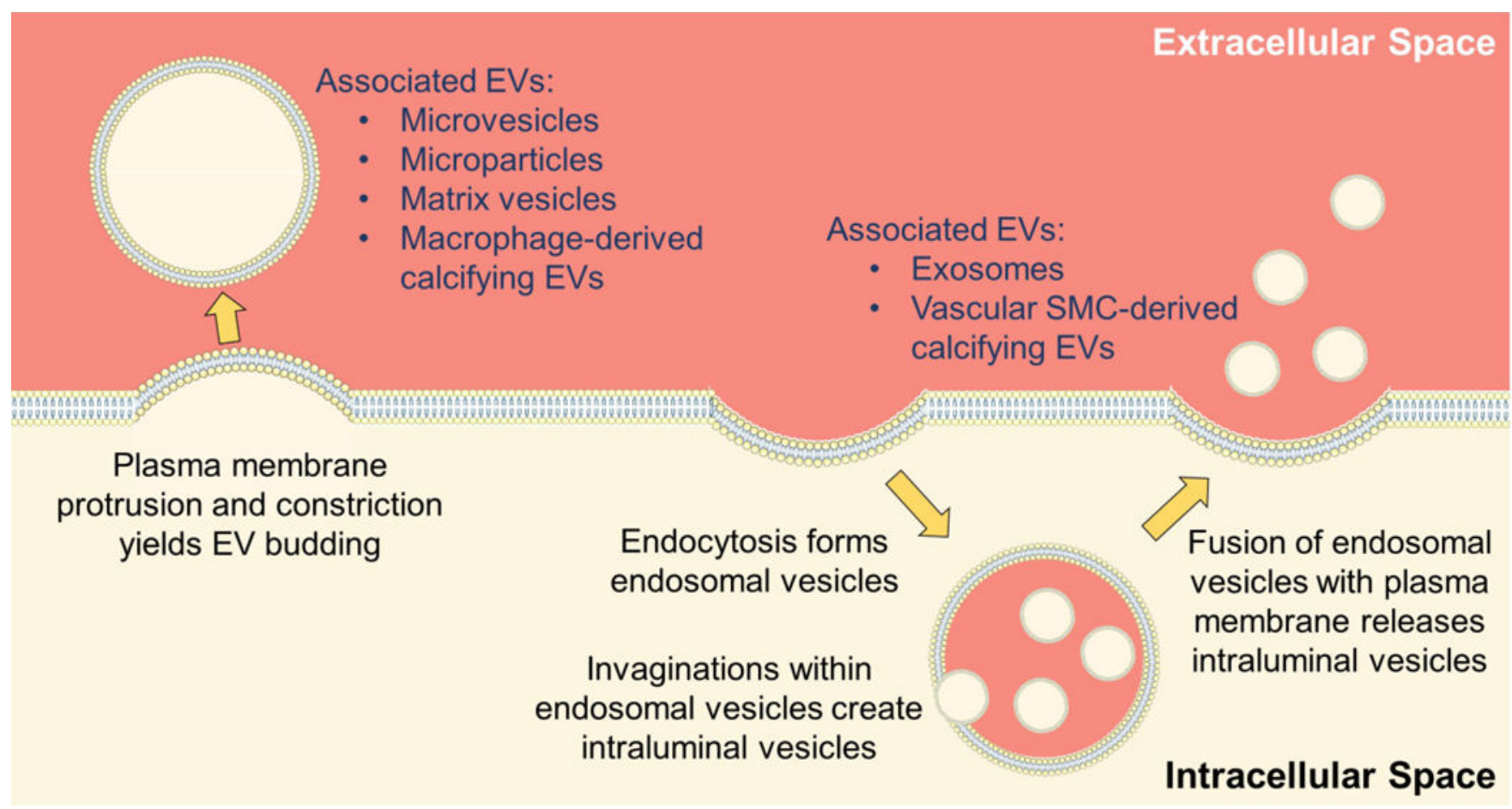

Figure 1.

EVs form from direct budding of the plasma membrane or through intracellular trafficking mechanisms. Figure created using Servier Medical Art images (http://smart.servier.com). 


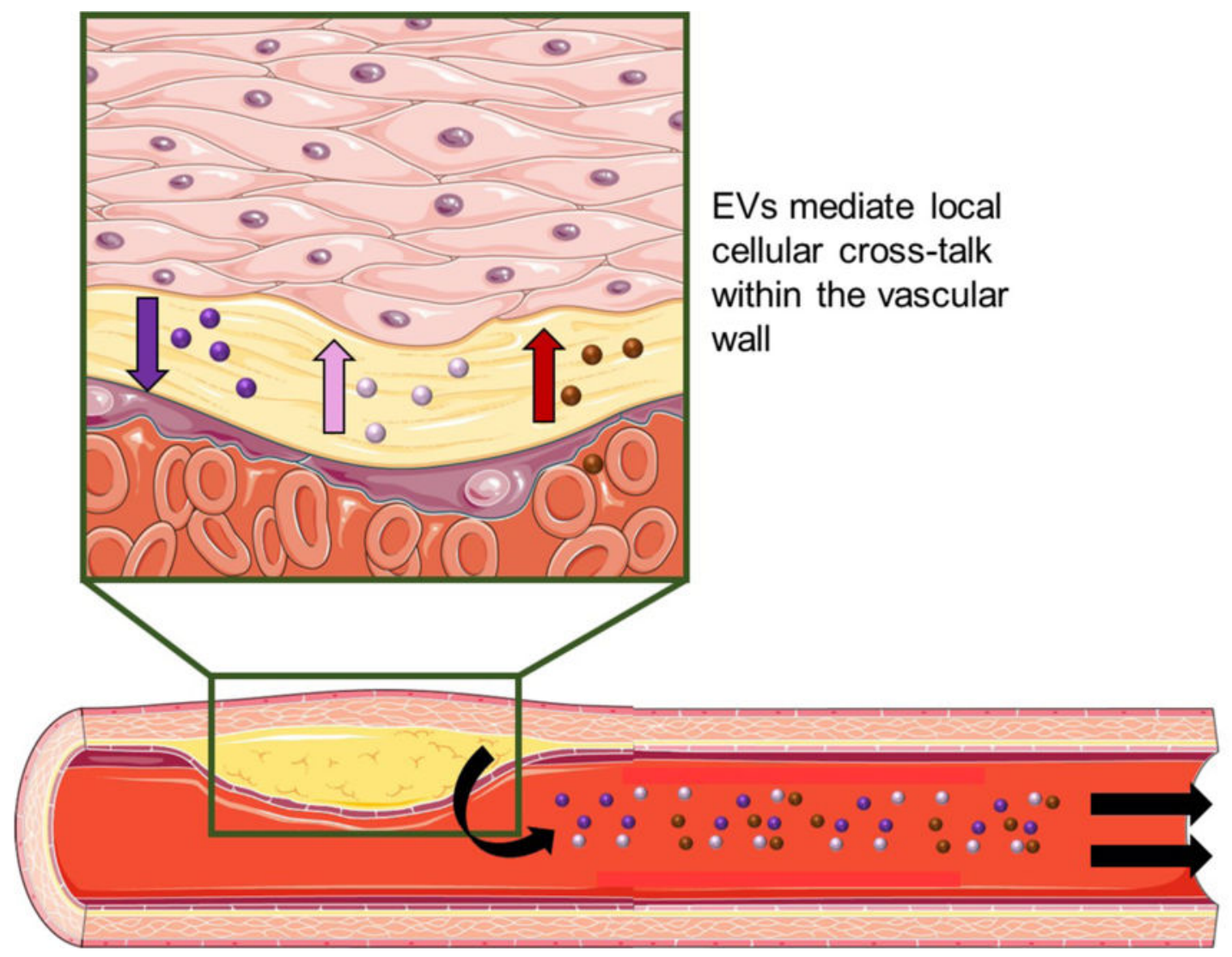

Circulating EVs mediate cargo transfer between tissues

Figure 2.

EVs can transfer cargo between cells in the vascular wall, mediating local cellular cross-talk. A portion of the local EVs may also enter the bloodstream and carry transfer cargo to downstream targets. Figure created using Servier Medical Art images (http:// smart.servier.com). 

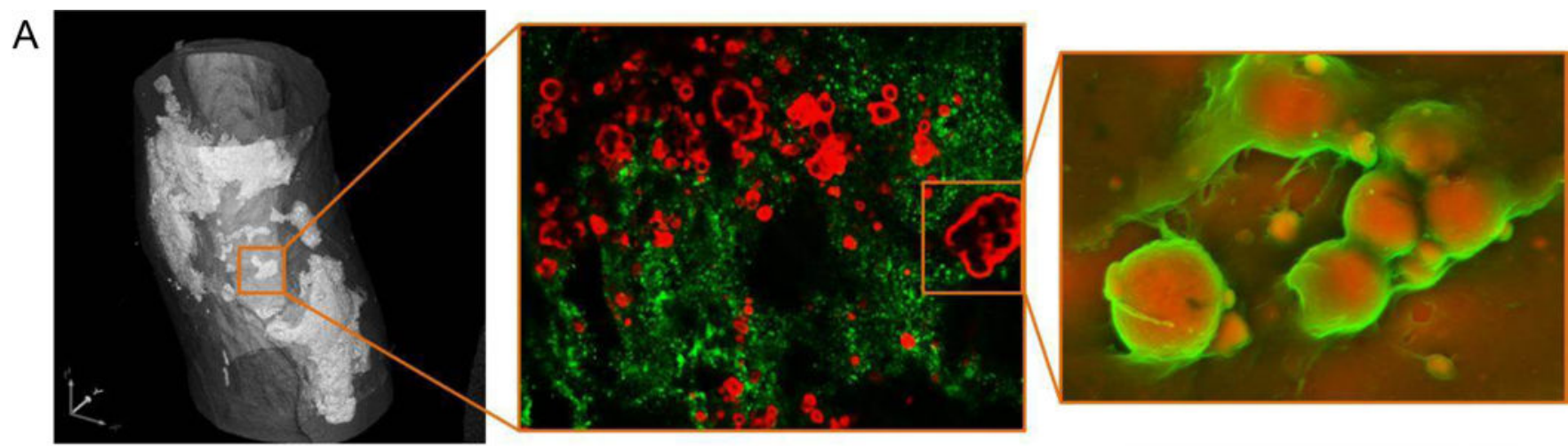

B
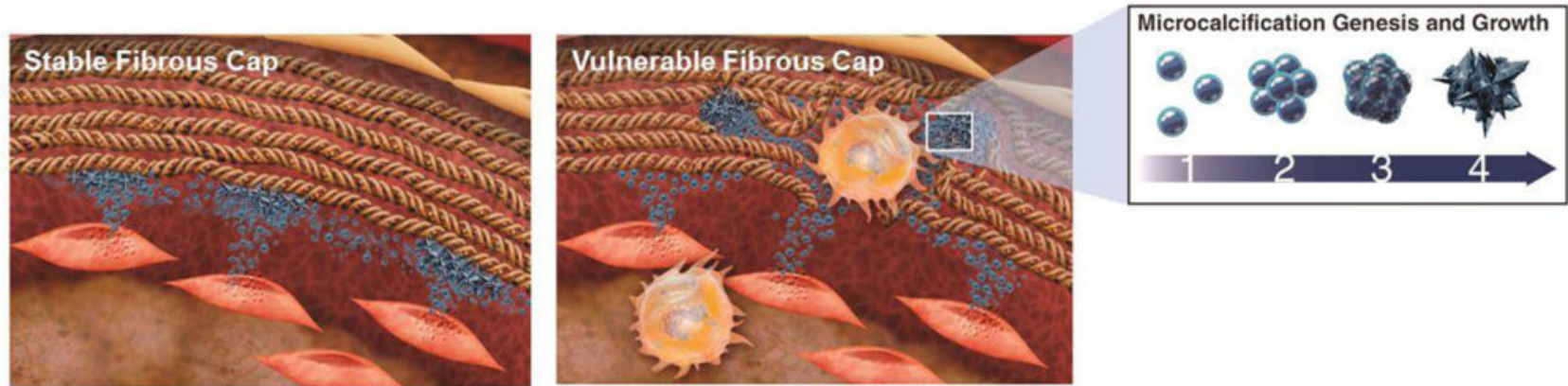

Figure 3.

Calcific mineral forms from merger of calcifying EVs. A) Microcalcifications are observed in high resolution micro-computed tomography. Fluorescence microscopy shows microcalcifications (red) between collagen fibers (green), and electron microscopy reveals that the microcalcifications are composed of aggregated EVs. B) Collagen acts as a scaffold that directs the formation of calcification either as large calcifications beneath collagen-rich stable fibrous caps or as microcalcifications between degraded collagen fibers in vulnerable caps. Mature calcifications occur when EVs accumulate, aggregate, merge, and nucleate hydroxyapatite. Adapted from [20]. 


\section{Table 1}

\section{Cardiovascular-related effects observed in recent studies associated with EVs}

\begin{tabular}{|l|c|c|}
\hline Cardiovascular Association or Effect & Source Cell/Tissue & Reference(s) \\
\hline Altered protein expression in circulating EVs in response to atherogenic remodeling & Endothelial Cells SMCs & {$[6-8]$} \\
\hline EVs mediate local endothelial cell dysfunction in aortic aneurysms & SMCs & {$[10]$} \\
\hline microRNAs in circulating EVs regulate systemic glucose tolerance & Adipose & {$[13]$} \\
\hline EVs serve as nucleating foci for calcific mineral in cardiovascular tissues & SMCs Valve Interstitial Cells & {$[20-23]$} \\
\hline Thrombin generation begins with factors carried by EVs & Platelets SMCs Endothelial Cells & {$[25-28]$} \\
\hline Injection of EVs collected from cultured cells induce cardioprotective or reparative effects & Stem Cells Cardiosphere Cells & {$[33-34]$} \\
\hline
\end{tabular}

